\title{
Origem Anômala de Artéria Coronária Esquerda do Tronco Pulmonar: Síndrome de Bland-White-Garland
}

\author{
Isamara Fernanda Bregagnollo¹, Gustavo Souza Oliveira', \\ Rossano Cesar Bonatto1, Fábio Cardoso Carvalho ${ }^{1}$
}

\section{RESUMO}

A origem anômala da artéria coronária esquerda a partir do tronco pulmonar, conhecida como síndrome de BlandWhite-Garland (BWG), é uma doença rara, que, habitualmente, leva à morte antes do primeiro ano de vida. Os autores relatam o caso de uma criança branca, com 2 anos e 6 meses de idade, portadora da síndrome de BWG, e revisam a apresentação clínica, a fisiopatologia, o diagnóstico e o tratamento desses pacientes.

DESCRITORES: Cardiopatias congênitas. Anomalias dos vasos coronários. Artéria pulmonar, anormalidades. Insuficiência cardíaca congestiva, etiologia.

A origem anômala da artéria coronária esquerda a partir do tronco pulmonar caracteriza a rara síndrome de Bland-White-Garland (BWG), que pode resultar da septação anormal do tronco arterioso em aorta e artéria pulmonar ou da persistência dos brotos pulmonares, com involução dos brotos aórticos que formarão as artérias coronárias ${ }^{1}$. Trata-se de uma má formação que afeta um em cada 300 mil nascidos vivos, correspondendo a $0,5 \%$ das doenças cardíacas congênitas ${ }^{2}$. Responde pela causa de morte nas crianças afetadas numa freqüência de $80 \%$ a $85 \%$ antes do primeiro ano de vida ${ }^{3}$. Atualmente, são reconhecidas duas formas:

1. Infantil: não há formação de circulação colateral ou, se ocorrer, é discreta. Corresponde a cerca de $80 \%$ a $85 \%$ dos casos. Os sintomas mais freqüentes são dispnéia, insuficiência cardíaca congestiva e dor torácica

\footnotetext{
Setor de Hemodinâmica do Hospital das Clínicas da Faculdade de Medicina de Botucatu - UNESP, Botucatu, SP.

Correspondência: Isamara Fernanda Bregagnollo. Rua Capitão Andrade, 567 - Bairro Alto - Botucatu, SP - CEP 18601-545

Tel.: (14) 3811-6121 • E-mail: isamara36@gmail.com

Recebido em: 26/7/2007 • Aceito em: 28/8/2007
}

SUMMARY

\author{
Abnormal Origin of the Left Coronary \\ Artery From Pulmonary Artery: \\ The Bland-White-Garland Syndrome
}

The abnormal origin of the left coronary artery (LCA) from the pulmonary artery, known as Bland-White-Garland Syndrome (BWG), is a rare disease that, usually, results in death within the first year of life. The authors report the case of a $2 \quad 1 / 2$ year old Caucasian child, with BWC Syndrome. A review of the clinical presentation, physiopathology, diagnostic methods and treatment of such patients is also presented.

DESCRIPTORS: Heart defects, congenital. Coronary vessel anomalies. Pulmonary artery, abnormalities. Heart failure, congestive, etiology.

aos esforços habituais do lactente, provocados por isquemia e disfunção ventricular.

2. Adulta: há formação de circulação colateral bem estabelecida. Corresponde de $15 \%$ a $20 \%$ dos casos. As principais manifestações incluem infarto agudo do miocárdio, insuficiências mitral e cardíaca congestiva, arritmias ventriculares e morte súbita ${ }^{1-3}$.

No presente trabalho, os autores relatam um caso da forma adulta e revisam a literatura e a fisiopatologia pertinentes.

\section{RELATO DO CASO}

Paciente TCP, branca, com 2 anos e 6 meses de idade, do sexo feminino, assintomática. Ao exame físico, durante avaliação pediátrica de rotina, apresentava-se com bom estado geral, acianótica, eupnéica, afebril, com mucosas úmidas e coradas, e todos os pulsos palpáveis, rítmicos, simétricos e com amplitude preservada. Apresentava peso de $12,3 \mathrm{~kg}$, estatura de $88 \mathrm{~cm}$, índice de massa corpórea de 15,9 kg/m², freqüência cardíaca de 110 bpm, freqüência respiratória de 23 ipm, pressão arterial de 96 × $60 \mathrm{mmHg}$, e pulmões com expansibilidade preservada, sem ruídos adventícios e evidências de derrame pleural. 
Bregagnollo IF, et al. Origem Anômala de Artéria Coronária Esquerda do Tronco Pulmonar: Síndrome de Bland-White-Garland. Rev Bras Cardiol Invas 2007; 15(4): 438-442.

Ao exame cardiológico, apresentou ictus visível, palpável e impulsivo (+++) no 6으 espaço intercostal esquerdo (EIE), $3 \mathrm{~cm}$ fora da linha hemiclavicular, rítmico. Sem frêmitos ou bulhas palpáveis. Bulhas rítmicas e hipofonéticas. Sopro sistólico $2+/ 4+$, rude, mesossistólico, audível em foco mitral e irradiado para axila esquerda. O abdome apresentava ruídos hidroaéreos normais, sem evidências de visceromegalias. Os membros inferiores apresentavam-se sem edema. À eletrocardiografia, foram observados ritmo sinusal, sobrecarga de câmaras esquerdas, e alteração da repolarização ventricular sugestiva de isquemia em parede anterior extensa. À radiografia de tórax, foi observado índice cardiotorácico de 0,66, com trama vascular pulmonar normal e aumento de câmaras esquerdas (Figura 1). À ecocardiografia, foram observados dilatação de câmaras cardíacas esquerdas, hipocinesia ântero-apical, refluxo mitral moderado e dilatação da artéria coronária direita, além de não ter sido possível a visualização da coronária esquerda (Figura 2). Ao estudo hemodinâmico, foram observadas as seguintes pressões, em mmHg: ventrículo esquerdo, 101/16; aorta, 102/59; tronco pulmonar, 35/19/25; ventrículo direito, 37/8; e átrio direito, 8. À aortografia, foram observadas valva aórtica bicúspide e competente e artéria coronária direita dilatada, não sendo possível a visibilização da artéria coronária esquerda. O ventrículo esquerdo apresentava-se com aumento dos volumes sistólico e diastólico finais e refluxo mitral moderado, e o átrio esquerdo estava dilatado. A angiografia seletiva de coronária direita evidenciou artéria coronária direita dilatada, tortuosa e com ampla circulação colateral para coronária esquerda, cujo tronco tinha origem na artéria pulmonar. As artérias descendente anterior e circunflexa apresentavam bom calibre e posição anatômica preservada (Figura 3).

\section{DISCUSSÃO}

$\mathrm{Na}$ síndrome de BWG relatada pelos autores, o mecanismo de agressão ocorre por um crônico processo de isquemia miocárdica. Inicialmente, há vasodilatação coronária, em uma tentativa de melhora da isquemia; posteriormente, o organismo procura compensar a baixa oxigenação por meio de circulação colateral, que provém da artéria coronária direita. Dessa forma, estabelece um fluxo sanguíneo do sistema aórtico de alta pressão para o sistema pulmonar de baixa pressão, caracterizando fluxo sanguíneo aortopulmonar através das artérias coronárias. A isquemia miocárdica crônica leva a um processo de remodelação miocárdica, que, a curto prazo, mantém o desempenho cardíaco, mas que, a longo prazo, desencadeia o processo de remodelação cardíaca, o qual, se não interrompido, acarreta, em prazo variável, disfunção ventricular progressiva, insuficiência cardíaca e morte. Os sintomas mais freqüentes são: dispnéia, fadiga intensa, palidez, fácies de dor, infarto agudo do miocárdio, insuficiência mitral, arritmias e morte súbita. O quadro clínico e os exames complementares não-invasivos, muitas vezes, possibilitam o diagnóstico, sendo, no entanto, utiliza-

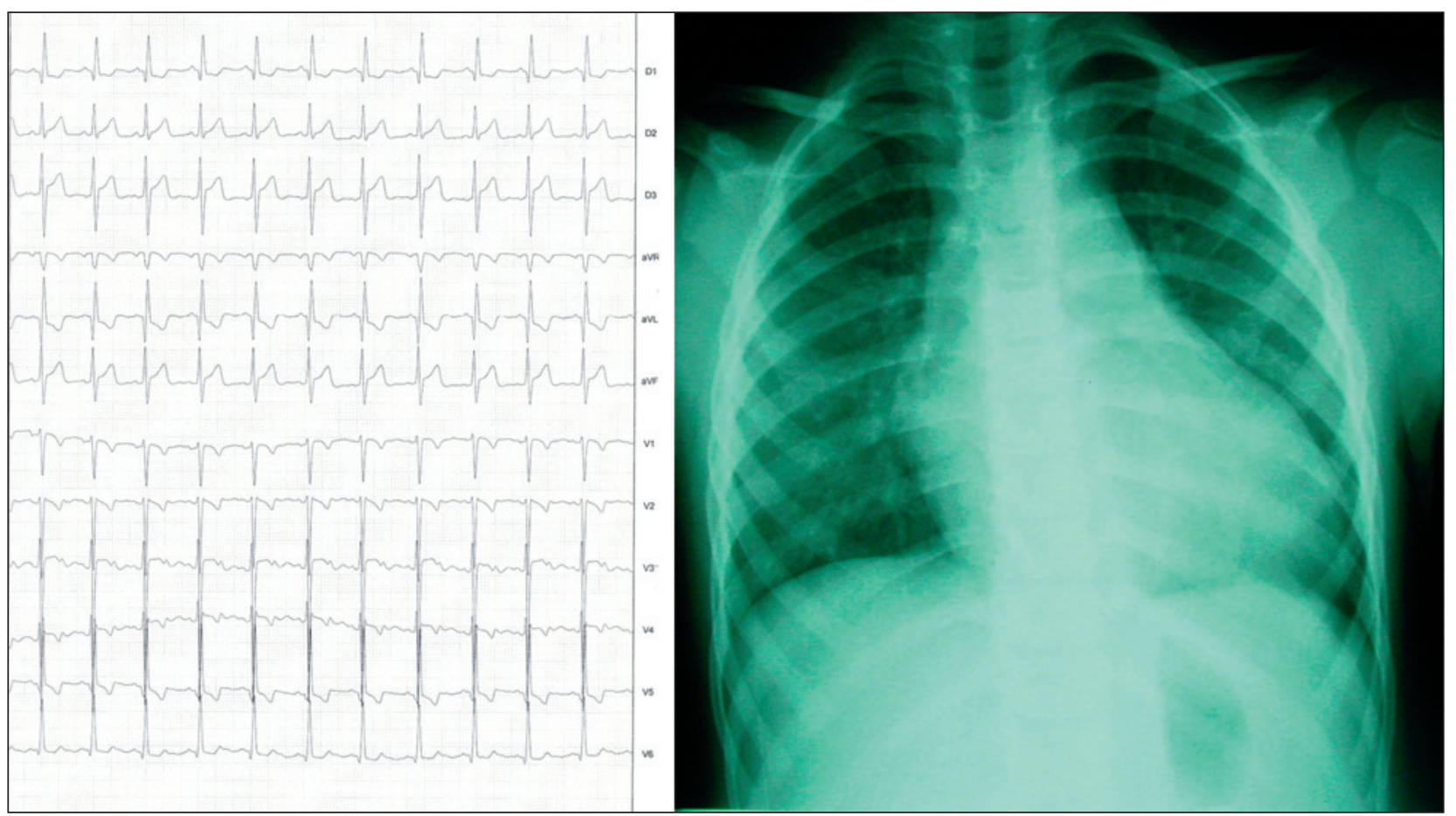

Figura 1 - Eletrocardiograma evidenciando taquicardia sinusal, sobrecarga de câmaras esquerdas e alterações difusas da repolarização ventricular, principalmente nas derivações precordiais. Radiografia de tórax demonstrando aumento de câmaras esquerdas. 


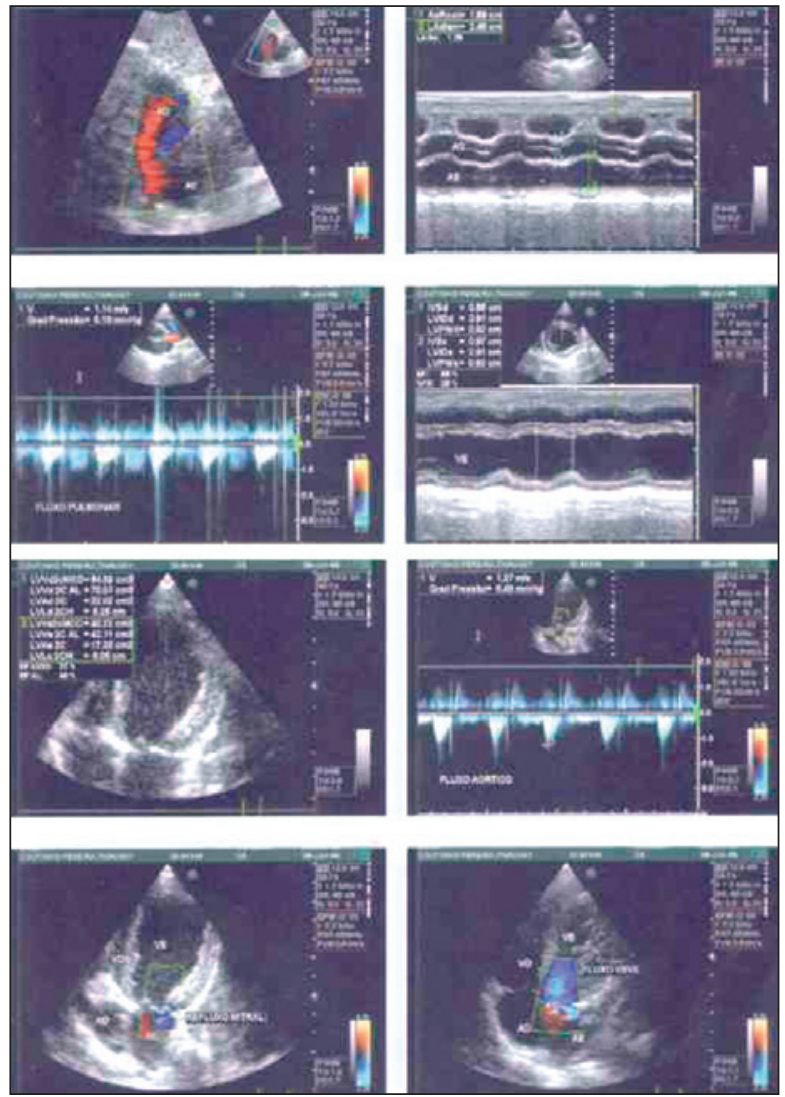

Figura 2 - Presença de refluxo ventrículo esquerdo-átrio esquerdo (VE-AE) moderado, aumento de câmaras esquerdas, hipocinesia de parede ântero-apical, fração de ejeção (FE) de $45 \%$.

do o cateterismo cardíaco para diagnóstico definitivo na escolha da abordagem terapêutica. O tratamento definitivo para a anomalia é cirúrgico. No caso descrito, esses exames possibilitaram caracterizar, detalhadamente, as alterações presentes nessa paciente.

A síndrome de BWG gera seqüelas nos pacientes acometidos, por um mecanismo de isquemia miocárdica, que se manifesta na vida extra-uterina ${ }^{1}$. Durante a vida intra-uterina, o sangue proveniente do tronco pulmonar possui alta saturação de oxigênio e a demanda miocárdica pelo mesmo é alta. Na vida extrauterina, pela organização da circulação, o sangue que chega à artéria coronária esquerda passa a apresentar baixa saturação de oxigênio ${ }^{3}$. Concomitantemente, as necessidades metabólicas do recém-nascido aumentam, elevando também o consumo de oxigênio miocárdico. Como conseqüência, forma-se, no decorrer do tempo, uma circulação colateral proveniente da artéria coronária direita e de seus ramos, com o intuito de complementar a irrigação miocárdica ineficaz da artéria coronária esquerda ${ }^{2,3}$. No princípio, essa circulação colateral é pouco desenvolvida e o ventrículo esquerdo sofre um crônico processo de isquemia, respondendo com vasodilatação coronária e redução da resistência ao fluxo, para preservar a oferta de oxigênio adequada às demandas metabólicas ${ }^{1}$. Em breve a reserva de fluxo coronário se esgota, dando início a um processo crescente de isquemia, com conseqüente disfunção ventricular. O processo de desenvolvimento de circulação colateral continua, com aumento do calibre e do número dos vasos colaterais; porém, como a artéria coronária esquerda está conectada a um sistema de baixa pressão, o sangue tende a fluir retrogradamente pela mesma, até atingir a artéria pulmonar, desviando o fluxo dos vasos miocárdicos, que possuem maior resistência. Isso constitui o chamado roubo pulmonar-coronário ou shunt coronária direita para a esquerda ${ }^{1,3}$. Nessas circunstâncias, instala-se um quadro de isquemia miocárdica crônica dos territórios irrigados pelas artérias coronárias esquerda e direita. Em conseqüência, ocorre disfunção ventricular esquerda tanto sistólica como diastólica, com dilatação ventricular progressiva. O processo de remodelação, que, a curto prazo, pode manter o desempenho cardíaco, a longo prazo promove alterações da função dos miócitos, da constituição do miocárdio e da geometria da cavidade ventricular esquerda ${ }^{4,5}$. As alterações da função dos miócitos e da composição do miocárdio comprometem a contratilidade e, conseqüentemente, o desempenho global do coração ${ }^{4,5}$. Atualmente, existem também evidências de que as alterações da geometria da câmara ventricular esquerda acarretam várias desvantagens hemodinâmicas para o ventrículo esquerdo. Uma das primeiras observações é que o ventrículo remodelado não somente aumenta as dimensões, mas também se torna mais esférico. A transformação da forma elíptica para esférica cria várias desvantagens mecânicas para a câmara, entre as quais se destacam: aumento do estresse parietal tanto sistólico como diastólico, desajuste da pós-carga, hipoperfusão subendocárdica, aumento do consumo de oxigênio miocárdico, manutenção da sobrecarga hemodinâmica, e acentuação da ativação dos mecanismos de remodelação e compensatórios da insuficiência cardíaca ${ }^{6}$. O segundo problema importante é que o aumento da esfericidade compromete a função dos músculos papilares, por estiramento, e a dilatação do anel mitral, resultando em insuficiência mitral de grau variável ${ }^{7}$. A regurgitação mitral impõe sobrecarga volumétrica para o ventrículo esquerdo, contribuindo para acentuar a dilatação ventricular, instalando-se, assim, um círculo vicioso que tende a comprometer progressivamente a função global do coração, independentemente dos mediadores neuro-humorais responsáveis pela remodelação cardíaca ${ }^{1-3,7,8}$.

No paciente relatado, esses aspectos tornam-se evidentes nas avaliações ecocardiográfica e hemodinâmica, que demonstram: aumento das dimensões e remodelação esférica do ventrículo esquerdo, refluxo mitral moderado, e comprometimento do desempenho cardíaco. A angiografia das artérias coronárias evidencia claramente a contrastação precoce da artéria coronária esquerda por meio de ampla rede de colaterais, formando-se verdadeiro fluxo arteriovenoso da artéria 
Bregagnollo IF, et al. Origem Anômala de Artéria Coronária Esquerda do Tronco Pulmonar: Síndrome de Bland-White-Garland. Rev Bras Cardiol Invas 2007; 15(4): 438-442.

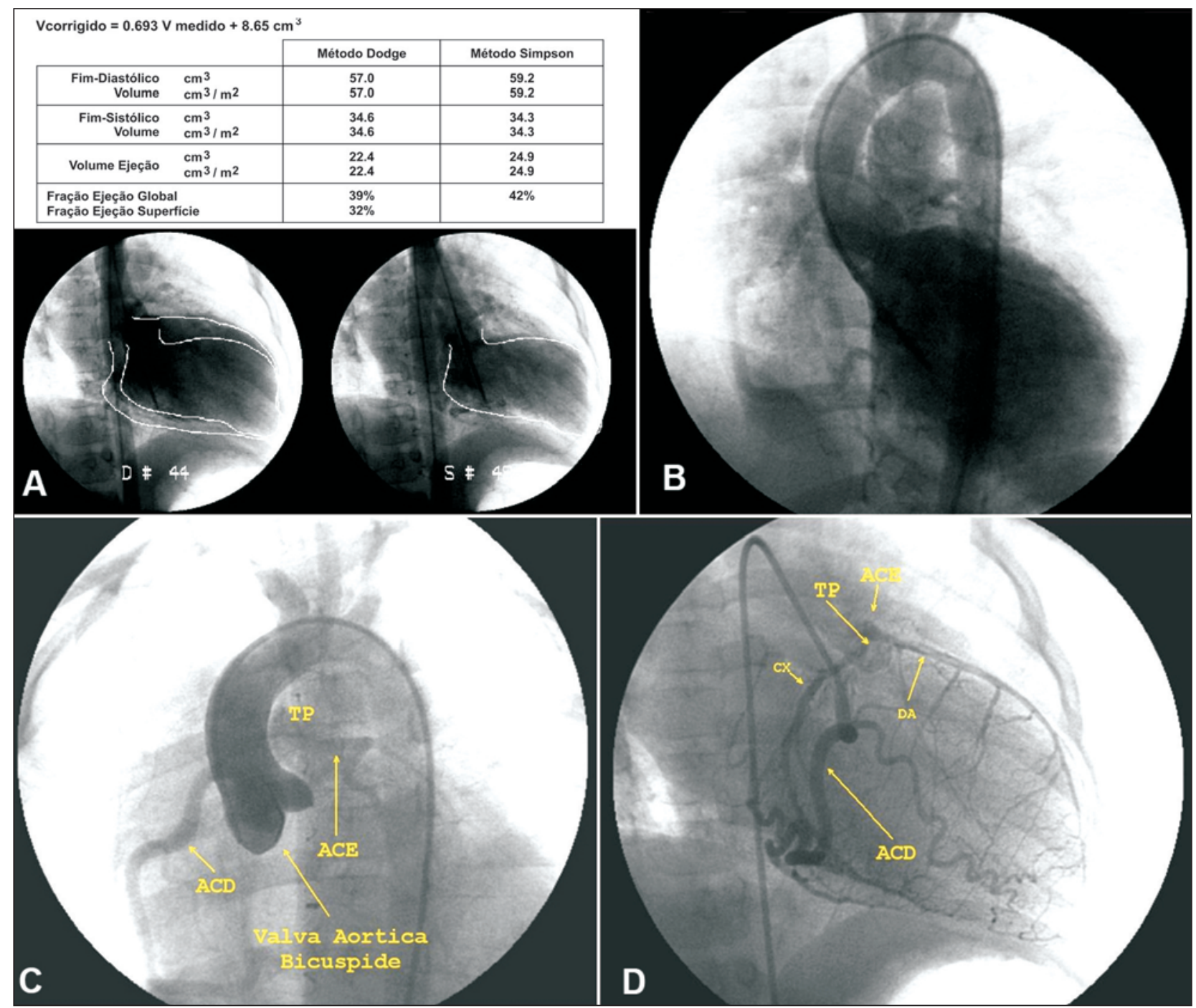

Figura 3 - A- Análise da função ventricular esquerda evidenciando discinesia ântero-apical e fração de ejeção (FE) de 42\%. B- Angiografia do ventrículo esquerdo com aumento do volume diastólico final. C- Aortografia com valva aórtica bicúspide, artéria coronária esquerda (ACE) originando-se do tronco da artéria pulmonar (TP) e artéria coronária direita (ACD) dilatada. D- ACD dilatada e tortuosa e ampla rede de colateral para a $\mathrm{ACE} . \mathrm{DA}=$ artéria descendente anterior; $\mathrm{CX}=$ artéria circunflexa.

coronária direita-artéria coronária esquerda-tronco pulmonar, que certamente promove isquemia miocárdica.

Os constantes episódios de isquemia levam à formação de pequenas áreas de necrose, o que pode acarretar insuficiências cardíaca e mitral. Esta última, por disfunção do músculo papilar e/ou dilatação do anel valvar mitral, foi observada na paciente descrito neste trabalho. No caso relatado, o ecocardiograma não permitiu a visualização da artéria coronária esquerda, sugerindo a presença de origem anômala desta. O cateterismo cardíaco confirmou o diagnóstico, revelou átrio e ventrículo esquerdos dilatados, disfunção da parede ântero-lateral do ventrículo esquerdo, regurgitação mitral, artéria coronária direita dilatada e presença de ampla rede de colaterais, com enchimento precoce da artéria coronária esquerda originando- se do tronco pulmonar ${ }^{9}$. O exame de escolha para o diagnóstico desses casos é a ecocardiografia bidimensional Doppler em cores, que, além de fazer o diagnóstico na quase totalidade dos casos, fornece informações valiosas e precisas sobre o grau de comprometimento miocárdico, a função global do coração e o estado anatomofuncional da valva mitral. No caso relatado, o estudo ecocardiográfico não foi suficiente para esclarecer adequadamente todas as alterações morfofuncionais para intervenção cirúrgica. Considerando-se que a instituição não dispunha de equipamento de angiotomografia e que havia necessidade de solucionar o caso com a maior brevidade possível, os autores optaram pela realização de estudo hemodinâmico com angiocoronariografia para estabelecer o diagnóstico definitivo e a programação cirúrgica ${ }^{1,9}$. 
O tratamento definitivo para a anomalia é cirúrgico $^{1,9,10}$. A cirurgia está sempre indicada e fica atrelada ao aparecimento de sintomas e/ou à presença de fluxo sanguíneo significativo através das artérias coronárias, pois ambos sinalizam grande risco de arritmias ventriculares e morte súbita, devendo ser realizada o mais precocemente possível ${ }^{1,2}$. Há diversas técnicas cirúrgicas que permitem a correção, mas não há estudos que apontem de forma clara qual a melhor. O que está bem estabelecido é o risco de morte cirúrgica, sabidamente mais elevado até os 18 a 24 meses de vida, devendo a correção cirúrgica, sempre que possível, ser realizada antes desse período ${ }^{1-3,9}$. A abordagem mais utilizada é o reimplante cirúrgico da artéria coronária esquerda anômala diretamente na aorta. Outras abordagens incluem: ligadura da artéria coronária esquerda no nível de sua origem na artéria pulmonar, revascularização com enxerto venoso ou artéria mamária interna, e cirurgia do túnel intrapulmonar (Takeuchi) ${ }^{1,2,10}$. A paciente deste relato foi submetida a cirurgia com reimplante da artéria coronária esquerda na aorta, com sucesso e boa evolução.

\section{REFERÊNCIAS BIBLIOGRÁFICAS}

1. Hoffman JIE. Congenital anomalies of the coronary vessels and the aortic root. In: Allen HD, Gutgesell HP, Clark EB, Driscoll DJ, eds. Moss and Adams' Heart disease in infants, children, and adolescents. Including the fetus and young adults. Baltimore:Williams \& Wikins;1995. p.769-91.
2. Barbetakis N, Efstathiou A, Efstathiou N, Papagiannopoulou $\mathrm{P}$, Soulountsi V, Fessatidis I. A long-term survivor of BlandWhite-Garland syndrome with systemic collateral supply: a case report and review of the literature. BMC Surg. 2005;5:23.

3. Nicholson WJ, Schuler B, Lerakis S, Helmy T. Anomalous origin of the coronary arteries from the pulmonary trunk in two separate patients with a review of the clinical implications and current treatment recommendations. Am J Med Sci. 2004;328(2):112-5.

4. Norton GR, Woodiwiss AJ, Gaasch WH, Mela T, Chung ES, Aurigemma GP, et al. Heart failure in pressure overload hypertrophy. The relative roles of ventricular remodeling and myocardial dysfunction. J Am Coll Cardiol. 2002;39(4): 664-71.

5. Nadal-Ginard B, Kajstura J, Leri A, Anversa P. Myocite death, growth, and regeneration in cardiac hypertrophy and failure. Circ Res. 2003;92(2):139-50.

6. Mann DL. Mechanisms and models in heart failure: a combinatorial approach. Circulation. 1999;100(9):999-1008.

7. Kono T, Sabbah HN, Rosman H, Alam M, Jafri S, Goldstein S. Left ventricular shape is the primary determinant of functional mitral regurgitation in heart failure. J Am Coll Cardiol. 1992;20(7):1594-8.

8. Houser SR, Margulies KB. Is depressed myocyte contractility centrally involved in heart failure? Circ Res. 2003;92(4):350-8.

9. Arce Casas A, Concheiro Guisán A, Cambra Lasaosa FJ, Pons Òdena M, Palomeque RA, Mortera PC. Isquemia coronária secundária a anomalias congênitas das artérias coronárias. An Pediatr. 2003;58(1):71-3.

10. Jacob JL, Salis FV. Origem anômala da artéria coronária esquerda do tronco pulmonar em mulher de 45 anos. Arq Bras Cardiol. 2003;81(2):196-8. 